\title{
Nanodiamond surface redox chemistry: influence of physicochemical properties on catalytic processes
}

\author{
Thomas S. Varley, ${ }^{*}$ Meetal Hirani, George Harrison \\ and Katherine B. Holt*
}

Received 17th March 2014, Accepted 18th March 2014

DOI: $10.1039 / c 4 f d 00041 b$

\begin{abstract}
Modification of an electrode with an immobilised layer of nanodiamond is found to significantly enhance the recorded currents for reversible oxidation of ferrocene methanol $(\mathrm{FcMeOH})$. Current enhancement is related to nanodiamond diameter, with enhancement increasing in the order $1000 \mathrm{~nm}<250 \mathrm{~nm}<100 \mathrm{~nm}<10 \mathrm{~nm}<5 \mathrm{~nm}$. We attribute the current enhancement to two catalytic processes: i) electron transfer between the solution redox species and redox-active groups on the nanodiamond surface; ii) electron transfer mediated by $\mathrm{FcMeOH}^{+}$adsorbed onto the nanodiamond surface. The first process is $\mathrm{pH}$ dependent as it depends on nanodiamond surface functionalities for which electron transfer is coupled to proton transfer. The adsorptionmediated process is observed most readily at slow scan rates and is due to selfexchange between adsorbed $\mathrm{FCMeOH}^{+}$and $\mathrm{FcMeOH}$ in solution. $\mathrm{FcMeOH}^{+}$has a strong electrostatic affinity for the nanodiamond surface, as confirmed by in situ infrared (IR) experiments.
\end{abstract}

\section{Introduction}

In recent years electrochemical experiments have been undertaken using electrodes composed of, or modified with, undoped nanodiamond materials. ${ }^{1}$ Given that bulk diamond is intrinsically insulating, with a band gap of $5.47 \mathrm{eV}$, it may seem unlikely that undoped diamond can perform as an electrode material. However, unsaturated bonding at the diamond surface and the presence of oxygen functionalities can give rise to surprisingly rich redox chemistry. ${ }^{2,3}$ For diamond nanomaterials the large surface atom to bulk atom ratio allows surface properties to dominate, hence nanodiamond is able to participate in electrochemical processes despite its insulating nature. Different types of nanodiamond with varying surface termination have been investigated for electrochemical activity. ${ }^{1-5}$ Most commonly used are detonation nanodiamond powders that have 
been used to construct cavity electrodes, ${ }^{5-7}$ sintered into pellet electrodes ${ }^{8}$ or dropcoated from solution onto electrodes. ${ }^{2,3,9}$ Detonation nanodiamond is synthesised by detonation of carbon-based explosives in the absence of oxygen, resulting in a carbonaceous soot from which the nanodiamond powder can be extracted and purified. ${ }^{10}$ The resulting powder is composed of relatively monodisperse diamond nanoparticles, with average diameters of 5 to $10 \mathrm{~nm}$, as shown in the TEM image in Fig. 1a. Detonation nanodiamond exhibits an array of surface carbon-oxygen functionalities (including ketones, esters, alcohols and acids) that have been characterised using FTIR $^{11,12}$ and XPS. ${ }^{13,14}$

Electrodes modified with an immobilised surface layer of detonation nanodiamond show an enhanced, catalytic electrochemical response towards various analytes such as nitrite, ${ }^{7}$ oxygen ${ }^{15}$ and haemoglobin, ${ }^{16}$ as well as common redox probes like $\mathrm{Ru}\left(\mathrm{NH}_{3}\right)_{6}{ }^{3+/ 2+}$ and $\mathrm{Fe}(\mathrm{CN})_{6}{ }^{3-/ 4-} .{ }^{2,3,9}$

It is proposed that surface functionalities, defect sites and unsaturated bonding give rise to surface electronic states with energies within the band gap of undoped diamond. ${ }^{3}$ It has been demonstrated that these surface states can act as both electron donors and acceptors and can support catalytic redox processes in the presence of specific redox-active molecules via a feedback mechanism. ${ }^{\mathbf{1 - 3 , 9}}$ This mechanism is illustrated schematically in Fig. 1b. Briefly, a species such as $\mathrm{FcMeOH}$ can undergo oxidation at an electrode to produce $\mathrm{FcMeOH}^{+}$. In the presence of nanodiamond, the $\mathrm{FcMeOH}^{+}$may then be reduced by redox active functionalities on the nanodiamond surface, resulting in regeneration of $\mathrm{FcMeOH}$. This continual regeneration of $\mathrm{FcMeOH}$ in the electrode diffusion layer results in an enhanced catalytic current. In situ IR studies of $5 \mathrm{~nm}$ diameter nanodiamond in the presence of redox probes have implicated surface quinoneand hydroquinone-like moieties in this redox mediation. ${ }^{11}$ Catalytic current enhancements at nanodiamond-modified electrodes, via the mechanism shown in Fig. 1b, have previously been reported for charged species such as $\mathrm{Ru}\left(\mathrm{NH}_{3}\right)_{6}{ }^{3+/ 2+}$ and $\mathrm{Fe}(\mathrm{CN})_{6}{ }^{3-/ 4-} .{ }^{3,9}$ At nanodiamond-modified electrodes, peak currents for these species increase $2-4$ fold over diffusion-controlled currents at the unmodified electrode. ${ }^{1-3,9}$ However, as the charge on the nanodiamond surface changes with the protonation state of the surface functionalities,

(a)

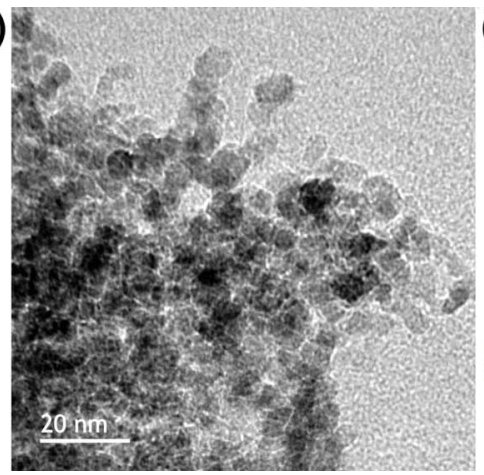

(b)

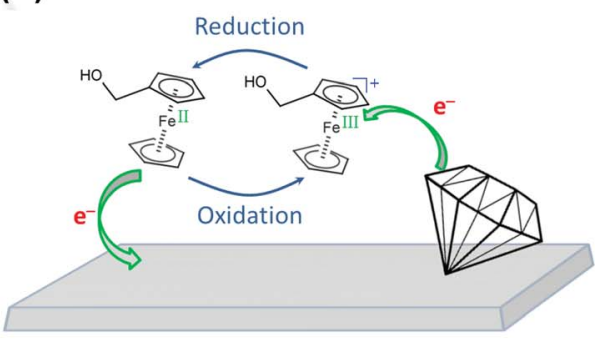

Catalytic feedback mechanism

Fig. 1 (a) TEM micrograph of $10 \mathrm{~nm}$ diameter nanodiamond. (b) Illustration of the nanodiamond feedback mechanism. The green arrows depict the direction of electron transfer. 
electrostatic interactions between the surface and redox probe must be taken into account when carrying out analysis.

In this paper we focus on nanodiamond materials of different size and morphology and their interaction with the outer-sphere and uncharged redox probe $\mathrm{FcMeOH}$. We find that electrodes modified with nanodiamond exhibit significantly enhanced catalytic currents for the reversible oxidation of this neutral species compared to charged redox species. We investigate the electrochemical response when changing the diameter of the nanodiamond particles used and the $\mathrm{pH}$ and ionic strength of the electrolyte solution. Analysing the effect of these physicochemical parameters, alongside factors such as redox species concentration and voltammetric scan rate, provides further insight into the mechanisms of current enhancement and nanodiamond surface properties.

\section{Experimental}

\subsection{Equipment}

All electrochemical experiments were carried out using a $\mu$ Autolab PGSTAT potentiostat (Eco Chemie, Utrecht, Netherlands) running GPES (v4.9), or a CHI910B Scanning Electrochemical Microscope/bipotentiostat ( $\mathrm{CH}$ Instruments, Inc. Austin, Texas, USA) running CHI (v6.28). Electrochemistry was performed using a three electrode setup. The working electrode was a boron-doped diamond disk $(d=3.0 \mathrm{~mm})$, press fitted into a PEEK body (Windsor Scientific, Slough, United Kingdom). The counter electrode was fabricated in-house from platinum wire and sheet, cut into flags of approximately $4 \mathrm{~cm}^{2}$. A stable reference potential was achieved by using an $\mathrm{Ag} / \mathrm{AgCl}$ (sat. $\mathrm{KCl}$ ) $\mathrm{RE}-5 \mathrm{~B}$ reference half-cell (BASi, West Lafayette, America).

A Carbolite MTF 1200 horizontal tube furnace was used to pre-treat and clean all nanodiamond samples. The temperature control unit and associated electronics were designed and built in house. Mid-infrared spectra were recorded (100 scans) in attenuated total reflectance (ATR) mode using a Bruker Tensor 27 Fourier transform infrared (FTIR) spectrophotometer (Bruker, Coventry, United Kingdom) fitted with a room temperature DLaTGS detector at $4 \mathrm{~cm}^{-1}$ resolution. The Platinum ATR accessory consisted of a diamond prism operating with one reflection. Background spectra were collected prior to each experiment (100 scans). Transmission electron micrograph (TEM) images were recorded using a Jeol JEM 2100 TEM with a $200 \mathrm{kV}$ accelerating voltage using a LaB6 filament. All nanoparticles were deposited from the same solutions used for modifying electrode surfaces. Carbon coated copper TEM grids were used as the nanoparticle support.

\subsection{Chemicals and solutions}

Nanodiamond (10 $\mathrm{nm}$ mean particle size, measured by TEM analysis, >97\%), potassium chloride (puriss grade, $>99.5 \%$ ), potassium dihydrogen phosphate $(>98 \%)$, potassium phosphate dibasic (puriss, $>99 \%$ ), potassium hexacyanoferrate trihydrate (98.5-102.0\%) and ferrocene methanol (97\%) were all purchased from Sigma-Aldrich (Dorset, England). Other nanodiamond samples were gifted by a collaborator. All solutions were prepared using water from a Millipore Milli-Q Gradient A10 water filter (18.2 M $\mathrm{cm}$ ). Electrodes were cleaned 
by mechanical polishing with MasterPrep alumina polishing suspension, $0.05 \mu \mathrm{m}$ (Buehler, Düsseldorf, Germany), and deionized water using 2-7/8" microcloth polishing pads (Buehler, Düsseldorf, Germany). Electrochemical measurements were carried out in either potassium chloride $(0.1 \mathrm{M})$ with a recorded $\mathrm{pH}$ of 5.88 , or a mixture of the phosphate buffer solutions potassium dihydrogen phosphate $(0.1 \mathrm{M})$ and potassium phosphate dibasic $(0.1 \mathrm{M})$ mixed in different proportions to achieve the required $\mathrm{pH}$.

All nanodiamond samples were treated in a furnace at $425^{\circ} \mathrm{C}$ for $4 \mathrm{~h}$ (including temperature ramp times, which were set at $10^{\circ} \mathrm{C} \mathrm{min}^{-1}$ ) in air. This was to remove any $\mathrm{sp}^{2}$ graphitic carbon from the surface and to maximise the number of oxygen terminating functional groups. ${ }^{17}$ Nanodiamond suspensions were prepared by adding nanodiamond to purified water, centrifuging twice (13 $200 \mathrm{rpm}$ for 10 $\mathrm{min}$ ) and briefly sonicating to ensure large aggregates were broken up. The mass per volume concentrations of the various nanodiamond solutions were: $5 \mathrm{~nm}$ ND $1.50 \mathrm{mg} \mathrm{ml}{ }^{-1} ; 10 \mathrm{~nm} \mathrm{ND} 1.50 \mathrm{mg} \mathrm{ml}^{-1} ; 100 \mathrm{~nm} \mathrm{ND} 1.55 \mathrm{mg} \mathrm{ml}^{-1} ; 250 \mathrm{~nm} \mathrm{ND}$ $1.60 \mathrm{mg} \mathrm{ml}^{-1}$ and $1000 \mathrm{~nm} \mathrm{ND} 1.7 \mathrm{mg} \mathrm{ml}^{-1}$.

\subsection{Preparation of nanodiamond-modified electrodes}

Nanodiamond films were drop coated onto clean, dry electrodes by pipetting the various nanodiamond suspensions $(2.5 \mu \mathrm{l})$ onto the electrode surface. The droplet was positioned and manoeuvred to ensure complete coverage of the electrode's surface. The nanodiamond coated electrode was left to air dry for 20 min and checked under an optical microscope to ensure even and complete coverage.

\section{Results}

\subsection{Characterisation of nanodiamond materials using FTIR and TEM}

Fig. 2 shows representative FTIR spectra and TEM micrographs for two of the nanodiamond samples used in this study: $5 \mathrm{~nm}$ detonation nanodiamond and $100 \mathrm{~nm}$ microcrystalline nanodiamond (produced by mechanical grinding of synthetic diamond). Two different detonation nanodiamond materials were studied and examination with TEM (Fig. 1a, Fig. 2b) revealed the powder to be composed of discrete spherical and relatively monodisperse particles; one sample with mean particle diameter $5 \mathrm{~nm}$ and another with a slightly larger particle diameter closer to $10 \mathrm{~nm}$. In both samples the individual nanoparticles aggregate together to form larger agglomerates. The surface of all of the nanodiamond samples used is highly oxidised due to the pretreatment procedure of heating in air, described in section 2.2 above. The FTIR spectrum of the $5 \mathrm{~nm}$ nanodiamond is complex, with a great number of different functionalities contributing to the vibrational spectrum. These functionalities have been characterised and discussed in detail previously, ${ }^{\mathbf{1 1} 12}$ and include alcohols and carbonyl species: $3393 \mathrm{~cm}^{-1}(\nu \mathrm{OH}), 2926 \mathrm{~cm}^{-1}\left(\nu \mathrm{CH}_{2}\right), 2847 \mathrm{~cm}^{-1}(\nu \mathrm{CH}), 1729 \mathrm{~cm}^{-1}(\nu \mathrm{C}=\mathrm{O})$ and $1634 \mathrm{~cm}^{-1}\left(\nu \mathrm{OH}_{\mathrm{Abs}}\right)$. The convoluted set of peaks between $1000-1500 \mathrm{~cm}^{-1}$ contains a mix of bending and stretching modes of various $\mathrm{C}-\mathrm{C}, \mathrm{C}-\mathrm{H}$ and $\mathrm{C}-\mathrm{O}$ containing functionalities. Of most interest to the present study is the presence of redox-active functionalities such as unsaturated ketone or 'quinone-like' moieties that can participate in redox cycling. ${ }^{11}$ 

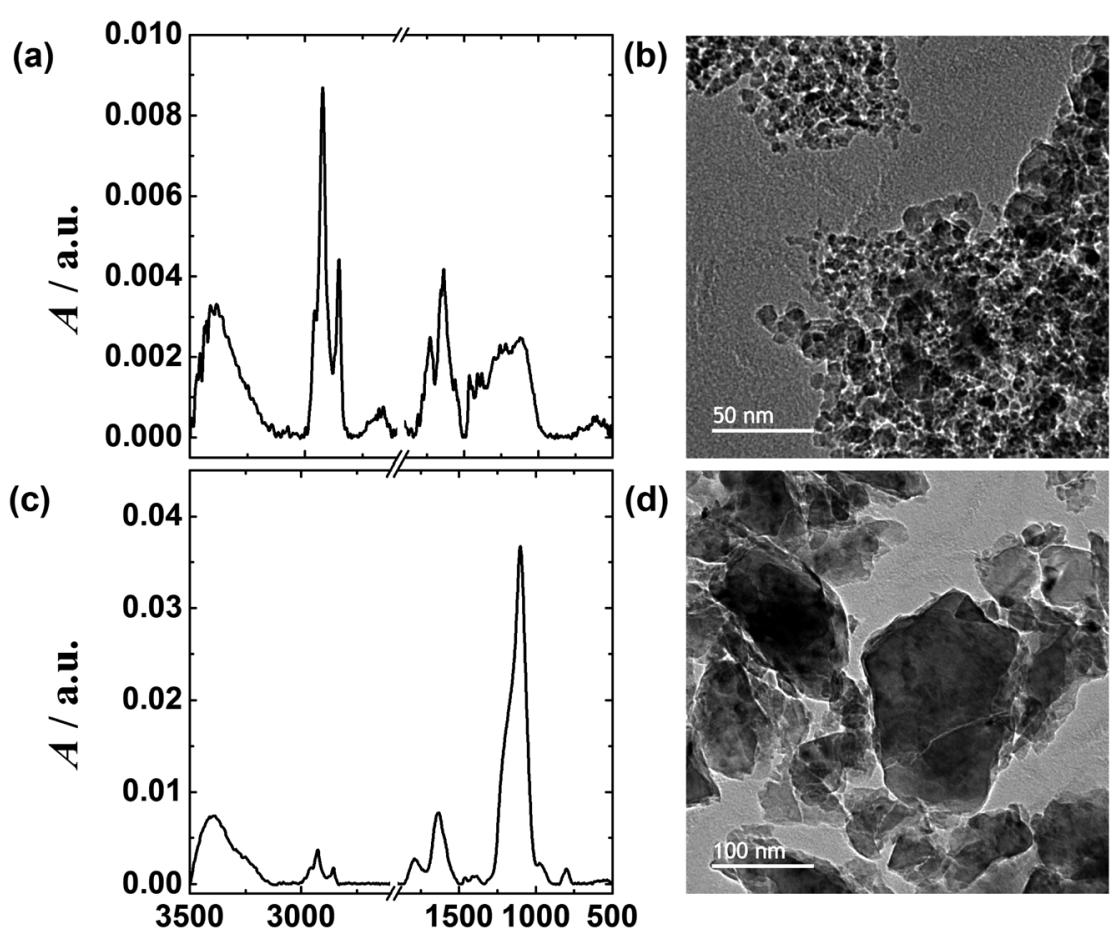

Fig. 2 ATR-FTIR spectra and TEM micrographs of $5 \mathrm{~nm}$, (a) and (b), and $100 \mathrm{~nm}$, (c) and (d), nanodiamond drop-cast films.

The 100, 250 and $1000 \mathrm{~nm}$ samples have pronounced crystal facets, consistent with nanodiamond produced through mechanical grinding of High Temperature High Pressure synthesis diamond..$^{18}$ Analysis of the size distribution of the $100 \mathrm{~nm}$ nanodiamond sample gives a mean particle size of $98 \mathrm{~nm}$ with a standard deviation of $35 \mathrm{~nm}$; the modal average size is $c a .100-120 \mathrm{~nm}$.

The crystalline nanodiamond samples all show a large absorption peak at $c a$. $1100 \mathrm{~cm}^{-1}$ in the IR spectra, attributable to nitrogen centres at defect sites within the bulk material. ${ }^{19}$ The IR spectra of these larger nanodiamonds also show absorption bands attributed to surface functionalities at $3394 \mathrm{~cm}^{-1}(\nu \mathrm{OH})$, $2923 \mathrm{~cm}^{-1}\left(\nu \mathrm{CH}_{2}\right), 2855 \mathrm{~cm}^{-1}(\nu \mathrm{CH}), 1790 \mathrm{~cm}^{-1}(\nu \mathrm{C}=\mathrm{O}), 1631 \mathrm{~cm}^{-1}\left(\nu \mathrm{OH}_{\mathrm{Abs}}\right)$ and $1103 \mathrm{~cm}^{-1}\left(\nu \mathrm{N}-\mathrm{V}^{-}\right)$, demonstrating the presence of similar surface functionalities to those on the detonation nanodiamond particles. Although the nanodiamond samples were produced by different synthetic methods, making direct comparison difficult, the oxidative pre-treatment results in similar surface oxygen functionalities, allowing us to anticipate similar electrochemical properties.

\subsection{Cyclic voltammetry (CV) investigations of nanodiamond-modified electrodes}

3.2.1 The effect of nanodiamond particle diameter on the voltammetric response of FCMeOH. Fig. 3a shows the cyclic voltammograms (CV) of $1 \mu \mathrm{M}$ FcMeOH at a clean boron doped diamond (BDD) electrode (black line) and a BDD electrode modified with $100 \mathrm{~nm}$ nanodiamond particles (red/blue lines). Both the 

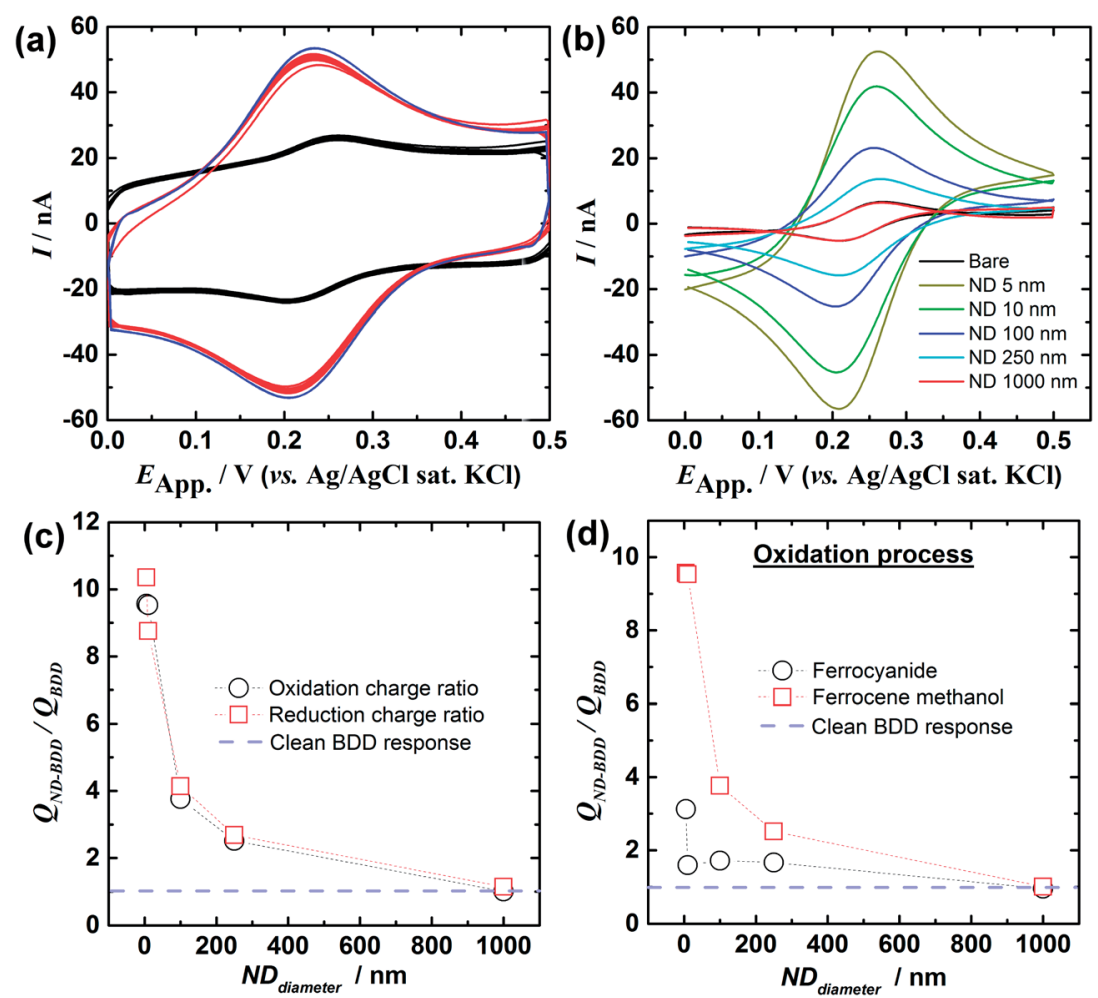

Fig. 3 (a) Voltammograms of FCMeOH $(1 \mu \mathrm{M})$ in $\mathrm{KCl}$ electrolyte solution $(0.1 \mathrm{M}, \mathrm{pH} 5.88)$ at a BDD (black) and $100 \mathrm{~nm}$ nanodiamond-modified BDD working electrode (red: first 10 scans, blue: final 10 scans, 100 scans total), $\nu=10 \mathrm{mV} \mathrm{s}^{-1}$. (b) Voltammograms (5 $5^{\text {th }}$ of scans) of FcMeOH (1 $\mu \mathrm{M})$ in $\mathrm{KCl}$ electrolyte solution (0.1 M, pH 5.88) on a BDD (black) and various different nanodiamond-modified BDD working electrodes (coloured lines, $d=5-$ $1000 \mathrm{~nm}), \nu=20 \mathrm{mV} \mathrm{s}^{-1}$, with background subtraction. (c) Charge ratio of the FcMeOH peak enhancement $\left(Q_{\mathrm{ND}-\mathrm{BDD}} / Q_{\mathrm{BDD}}\right)$ vs. nanodiamond size, calculated from data presented in (b). (d) Charge ratio of the $\mathrm{FCMeOH} / \mathrm{Fe}(\mathrm{CN})_{6}{ }^{4-}$ peak enhancement $\left(Q_{N D-B D D}: Q_{B D D}\right)$ vs. nanodiamond size calculated from data presented in (b) for FcMeOH and identical experiments carried out using $\mathrm{K}_{4} \mathrm{Fe}(\mathrm{CN})_{6}$. The blue dashed line highlights the charge ratio response for clean, un-modified BDD.

oxidation and reduction currents at the nanodiamond modified electrode show similar enhancements of $c a$. four times that of the clean BDD surface. This current enhancement is stable to repeated cycling over multiple scans. The peak separation $\Delta E_{\mathrm{p}}=\left|E_{\mathrm{p}}{ }^{\text {ox }}-E_{\mathrm{p}}{ }^{\text {red }}\right|$ is $\sim 30 \mathrm{mV}$ at the nanodiamond-modified electrode, in contrast to the theoretically predicted value of $59 \mathrm{mV}$ for a diffusioncontrolled one electron transfer. Fig. 3b shows CVs of FcMeOH at electrodes modified with nanodiamonds of different diameters.

The $1000 \mathrm{~nm}$ nanodiamond has a negligible effect on the electrochemical response, the $\mathrm{CV}$ being essentially identical to that at the clean BDD. Enhanced currents are observed at the other electrodes, with peak currents increasing in the order $250 \mathrm{~nm}<100 \mathrm{~nm}<10 \mathrm{~nm}<5 \mathrm{~nm}$. Oxidation and reduction peaks are enhanced equally in each case. The degree of current enhancement at the $10 \mathrm{~nm}$ and $5 \mathrm{~nm}$ nanodiamond-modified electrodes is quite dramatic. This has been 
quantified in Fig. 3c, where a 'charge enhancement ratio' has been calculated for each nanodiamond diameter. This is defined as the charge passed during oxidation or reduction at the nanodiamond-modified electrode divided by that passed at the clean BDD electrode under the same conditions, i.e. $Q_{\mathrm{ND}-\mathrm{BDD}} / Q_{\mathrm{BDD}}$. Charge passed during the oxidation of $\mathrm{FcMeOH}$ or reduction of $\mathrm{FcMeOH}^{+}$is increased by a factor of ten when the electrode is modified with a layer of $5 \mathrm{~nm}$ or $10 \mathrm{~nm}$ nanodiamond. A four-fold enhancement is noted for the $100 \mathrm{~nm}$ particles, falling to two-fold for the $250 \mathrm{~nm}$ nanodiamond, and no enhancement is seen for an electrode modified with $1000 \mathrm{~nm}$ particles.

In comparison to previous studies using the $\mathrm{Fe}(\mathrm{CN})_{6}{ }^{3-/ 4-}$ redox probe, ${ }^{9}$ the current enhancements for $\mathrm{FcMeOH}$ are considerably higher. This is shown in Fig. 3d, where the charge enhancement ratio for $\mathrm{Fe}(\mathrm{CN})_{6}{ }^{4-}$ oxidation is compared to that for $\mathrm{FcMeOH}$ oxidation at electrodes modified with different diameter nanodiamond. The data shows the same relationship between charge enhancement and nanodiamond diameter for both redox species, however, the magnitude of enhancement shows a strong dependence on the chemical nature of the redox species, with $\mathrm{FcMeOH}$ currents being significantly more enhanced.

3.2.2. The effect of the solution $\mathrm{pH}$ on the $\mathrm{CV}$ response of $\mathrm{FcMeOH}$ at nanodiamond-modified electrodes. CVs for $1 \mu \mathrm{M} \mathrm{FcMeOH}$ at clean BDD and 100 $\mathrm{nm}$ nanodiamond-modified BDD electrodes in different solutions of $\mathrm{pH}$ 5-9 are shown in Fig. 4a. At the BDD electrode the $\mathrm{CV}$ response of $\mathrm{FcMeOH}$ is independent of $\mathrm{pH}$, producing voltammograms with $\Delta E_{\mathrm{p}} c a .60 \mathrm{mV}$, consistent with oneelectron, reversible, diffusion-controlled electrochemistry. The currents are

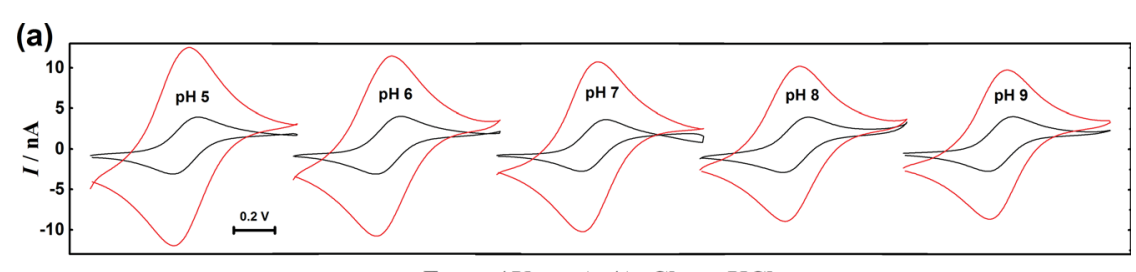

$E_{\text {App. }}$ / V (vs. Ag/AgCl sat. $\left.\mathrm{KCl}\right)$

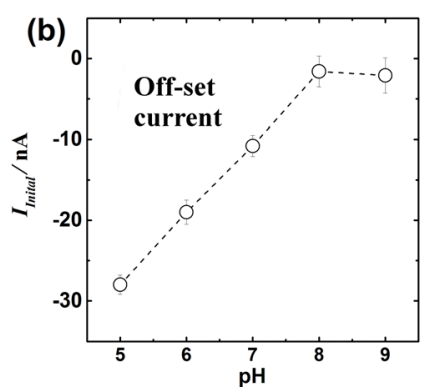

(c)

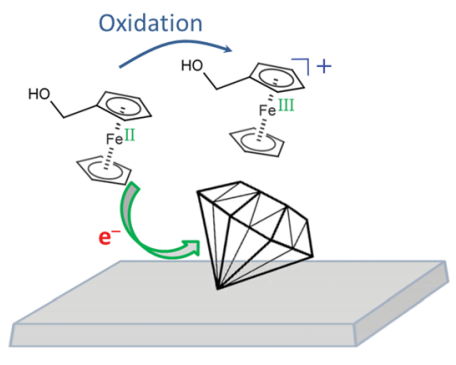

Fig. 4 (a) Cyclic voltammograms ( $1^{\text {st }}$ scan) of $\mathrm{FcMeOH}(1 \mu \mathrm{M})$ in aqueous solutions of $\mathrm{K}_{2} \mathrm{HPO}_{4}-\mathrm{KH}_{2} \mathrm{PO}_{4}(0.1 \mathrm{M})$ in various ratios to produce $\mathrm{pH}$ 5-9 electrolytes, at the clean BDD electrode (black) and nanodiamond modified BDD electrode (red), recorded at $5 \mathrm{mV} \mathrm{s}^{-1}$. (b) Initial off-set currents measured with different electrolytes of $\mathrm{pH} 5-9$ (data collected from the $1^{\text {st }}$ scan of the experiments displayed in (a)). (c) Illustration of FcMeOH oxidation performed by the nanodiamond. The green arrow depicts the direction of electron transfer. 
enhanced at the $100 \mathrm{~nm}$ nanodiamond-modified electrodes over all $\mathrm{pH}$ values, but the enhancement is $\mathrm{pH}$ dependent with $I_{\mathrm{p}}$ increasing as the $\mathrm{pH}$ is lowered. As for Fig. 3a, $\Delta E_{\mathrm{p}}$ for the nanodiamond-modified electrode is $\sim 30 \mathrm{mV}$ over the whole $\mathrm{pH}$ range. $I_{\mathrm{p}} v s$. $\nu$ analysis suggests mixed diffusion controlled and adsorption behaviour.

Each of the CVs at the nanodiamond-modified electrodes exhibits a non-zero 'off-set' reduction current at $0 \mathrm{~V}$. At this potential there should be no species in the solution available to be reduced (only $\mathrm{FcMeOH}$ is present and this can only be oxidised). We have reported this phenomenon previously for $5 \mathrm{~nm}$ nanodiamondmodified electrodes in solutions containing $\mathrm{Fe}(\mathrm{CN})_{6}{ }^{4-} \cdot{ }^{9}$ It is attributed in the present case to reduction of $\mathrm{FcMeOH}^{+}$species within the diffusion layer at the electrode surface. $\mathrm{FcMeOH}^{+}$is proposed to be generated spontaneously by the oxidation of $\mathrm{FcMeOH}$ at the nanodiamond surface (Fig. 4c). This oxidation takes place through electron exchange between nanodiamond surface functional groups, which are reduced concomitantly with the generation of $\mathrm{FcMeOH}^{+}$. The magnitude of the off-set current is $\mathrm{pH}$ dependent as shown in Fig. 4b. At pH 5, significant initial reduction currents are observed, but these become negligible at pH 8 and above.

3.2.3. The effect of solution ionic strength on the $\mathrm{CV}$ response of $\mathrm{FcMeOH}$ at nanodiamond-modified electrodes. The CVs for $1 \mu \mathrm{M} F \mathrm{FMeOH}$ at a $100 \mathrm{~nm}$ nanodiamond-modified BDD electrode in electrolytes of varying ionic strength are shown in Fig. 5. The ionic strength was varied by altering the concentration of the $\mathrm{KCl}$ electrolyte across five orders of magnitude. As the ionic strength is decreased, a pre-peak emerges from the oxidation wave, which shifts to more negative potentials with decreasing ionic strength (increasing the pre-peak to main-peak separation). The height of the main oxidation wave is decreased with decreasing ionic strength, while that of the reduction peak increases. In CV experiments the emergence of a pre-peak to an oxidation peak is usually taken as evidence of outer-sphere adsorption of the oxidation product at the electrode
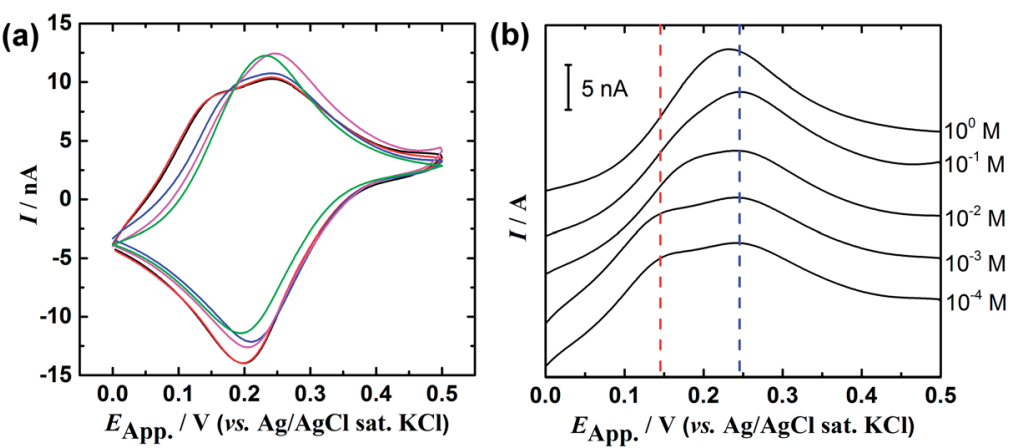

Fig. 5 (a) Cyclic voltammograms ( $5^{\text {th }}$ scan) of FCMeOH $(1 \mu \mathrm{M})$ in aqueous solutions containing various concentrations of $\mathrm{KCl}$ (black $=10^{-4} \mathrm{M}$, red $=10^{-3} \mathrm{M}$, blue $=10^{-2} \mathrm{M}$, magenta $=10^{-1} \mathrm{M}$, green $\left.=10^{\circ} \mathrm{M}, \mathrm{pH}=5.06-6.12\right)$ at a nanodiamond modified $(100 \mathrm{~nm})$ BDD working electrode, recorded at $5 \mathrm{mV} \mathrm{s}^{-1}$. (b) Stacked plot of the oxidation process only. The blue dashed line identifies the standard FcMeOH diffusion controlled peak (as seen for the clean BDD electrode), whereas the red dashed line highlights the emerging pre-peak. 
surface. In this case, the oxidation product $\mathrm{FcMeOH}^{+}$is likely to experience a positive electrostatic interaction with the negatively charged nanodiamond surface. ${ }^{20,21}$ Decreasing the ionic strength of the medium will increase the extent of adsorption, as the length to which the electric field of a surface can be felt is inversely proportional to the concentration of electrolyte (as described by DLVO theory). ${ }^{22,23}$ This is consistent with the observations in Fig. 5b, where the adsorption pre-peak can be seen to become more prominent as ionic strength is decreased and hence more $\mathrm{FcMeOH}^{+}$adsorbs onto the nanodiamond surface.

3.2.4. The effect of $\mathrm{CV}$ scan rate on the $\mathrm{CV}$ response of $\mathrm{FcMeOH}$ at nanodiamond-modified electrodes. The studies described in this section were carried out using $5 \mathrm{~nm}$ nanodiamond modified electrodes, as these showed the most extreme current enhancements. CVs were carried out in $1 \mu \mathrm{M}$ and $10 \mu \mathrm{M} \mathrm{FcMeOH}$ over scan rates of $1-500 \mathrm{mV} \mathrm{s}^{-1}$ (shown in Fig. 6, where currents have been normalised for clarity). Below scan rates of $10 \mathrm{mV} \mathrm{s}^{-1}$, two oxidation waves are clearly observed; one wave is positioned at the same $E_{\mathrm{p}}$ as the diffusion-controlled oxidation peak at the clean BDD electrode, and the other is a pre-peak to this main wave. A pre-peak to the reduction wave is also observed. As the scan rate is increased the two waves merge to form one broad peak located midway between the pre-peak and main peak positions. At scan rates above $c a .100 \mathrm{mV} \mathrm{s}^{-1}$ only one peak is observed, at the position expected for a simple diffusion-controlled mechanism. However, at very low concentrations of FcMeOH $(1 \mu \mathrm{M})$, at the faster scan rates, this peak becomes a steady state current response, indicating that oxidation is no longer taking place under diffusion-control.

\subsection{In situ ATR-IR spectroscopy of $5 \mathrm{~nm}$ nanodiamond in the presence of FcMeOH}

The electrochemical data is strongly suggestive of adsorption, of the oxidation product $\mathrm{FcMeOH}^{+}$in particular, at the nanodiamond surface. In situ ATR-FTIR spectroscopy experiments were therefore performed to monitor changes in the IR
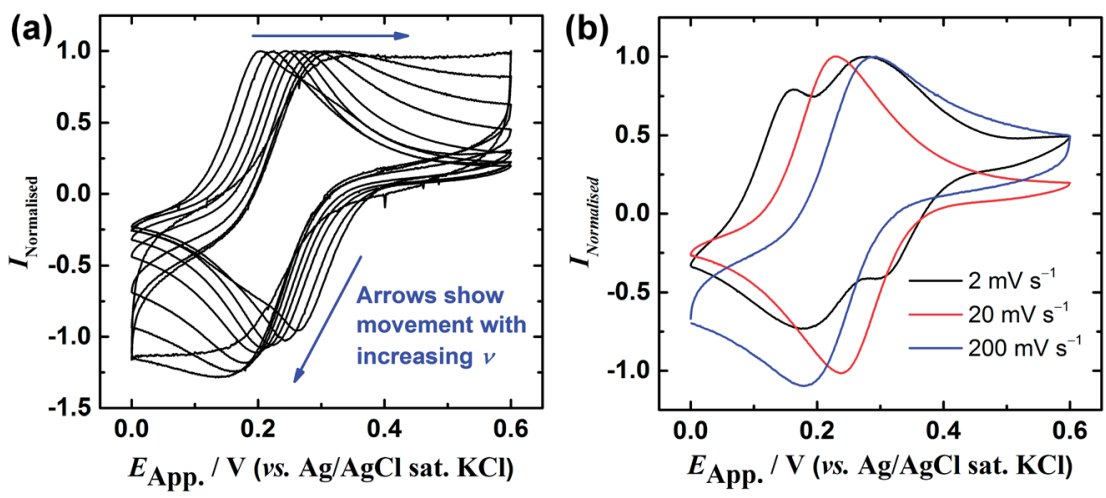

Fig. 6 (a) Normalised current (by max.) voltammograms of FcMeOH $(1 \mu \mathrm{M})$ for scan rates of $1,2,510,20,50,100,200$ and $500 \mathrm{mV} \mathrm{s}^{-1}$. (b) Voltammograms of FcMeOH $(10 \mu \mathrm{M})$ for scan rates of 2, 20 and $200 \mathrm{mV} \mathrm{s}^{-1}$. Experiments were performed with a $300 \mathrm{~s}$ preexperiment equilibration time (no applied potential) in $\mathrm{KCl}$ electrolyte solution $(0.1 \mathrm{M}, \mathrm{pH}$ 5.88 ) at a nanodiamond-modified ( $5 \mathrm{~nm}$ ) BDD electrode. 
absorption at the nanodiamond/solution interface in solutions containing FcMeOH or $\mathrm{FcMeOH}^{+}$. The experiments were performed by drop-casting a $5 \mathrm{~nm}$ nanodiamond film onto the ATR crystal. An enclosed liquid cell was clamped over the nanodiamond modified ATR crystal and gently filled with water to solvate the nanodiamond layer. After allowing time to equilibrate, a small volume of $\mathrm{FcMeOH}$ or $\mathrm{FcMeOH}^{+}$solution was gently introduced to the cell, and the FTIR spectrum between $3500-500 \mathrm{~cm}^{-1}$ was recorded over $120 \mathrm{~min}$. Fig. 7 shows the IR difference spectra obtained after 120 min of nanodiamond film immersion in the FcMeOH or $\mathrm{FcMeOH}^{+}$solution, along with a spectrum of the solid FcMeOH for comparison.

In the absence of nanodiamond, there is negligible change over $120 \mathrm{~min}$ in the IR spectra of the ATR crystal in FcMeOH solution. With the nanodiamond film, the presence of $\mathrm{FcMeOH}$ produces increases in infrared absorption at $c a$. 3200$3300 \mathrm{~cm}^{-1}(\nu \mathrm{OH})$ and $1650 \mathrm{~cm}^{-1}(\delta \mathrm{OH})$, i.e. predominantly in regions associated with $\mathrm{OH}$ vibrational modes. There are a greater number of changes observed for the nanodiamond film exposed to $\mathrm{FcMeOH}^{+}$solution. These occur in regions associated with $\mathrm{OH}, \mathrm{C}-\mathrm{H}$ and $\mathrm{C}-\mathrm{O}$ stretches and vibrations: $2925 \mathrm{~cm}^{-1}\left(\nu \mathrm{CH}_{2}\right.$, asym.), $2845 \mathrm{~cm}^{-1}\left(\nu \mathrm{CH}_{2}\right.$, asym.), $1648 \mathrm{~cm}^{-1}(\delta \mathrm{OH}), 1576-1393 \mathrm{~cm}^{-1}\left(\delta / \omega \mathrm{CH}_{2}\right)$ and $1044(\nu \mathrm{C}-\mathrm{C}-\mathrm{O})$, showing many features in common with the solid $\mathrm{FcMeOH}$ spectrum. $^{24}$

\section{Discussion}

\subsection{Mechanism of the catalytic current enhancement}

With the exception of $1000 \mathrm{~nm}$ nanodiamond, modification of the BDD electrode with an immobilised layer of nanodiamond results in enhanced CV currents for the reversible oxidation of $\mathrm{FcMeOH}$. Catalytic current enhancements have previously been reported for the redox probes $\mathrm{Fe}(\mathrm{CN})_{6}{ }^{3-/ 4-}, \mathrm{IrCl}_{6}{ }^{2-/ 3-}$ and $\mathrm{Ru}\left(\mathrm{NH}_{3}\right)_{6}{ }^{2+/ 3+}$, among others; $;^{1-3,6,9}$ however, currents achieved here for the neutral FcMeOH species are considerably higher than those observed for charged redox molecules. The current enhancement is partly attributed to a catalytic feedback mechanism, as shown in Fig. 1b. In this scheme, FcMeOH undergoes oxidation at

(a)

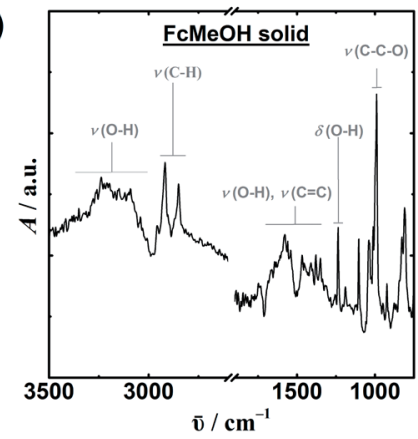

(b)

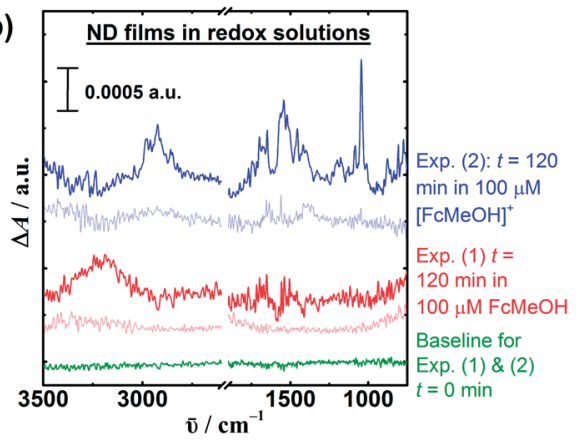

Fig. 7 (a) ATR-FTIR spectrum of FCMeOH (solid powder, black line), and (b) ATR-FTIR difference spectra (coloured lines) recorded for solutions of $\mathrm{FCMeOH}$ (red) and $\mathrm{FcMeOH}{ }^{+}$ (blue) on a clean ATR prism (transparent line), and in the presence of a nanodiamond dropcast film (solid line). All aqueous solutions contained $\mathrm{KCl}(0.1 \mathrm{M}, \mathrm{pH} 5.88$ ). 
the $\mathrm{BDD}$ electrode to produce $\mathrm{FcMeOH}+$. $\mathrm{FcMeOH}$ is then regenerated rapidly by the reduction of $\mathrm{FcMeOH}^{+}$at the nanodiamond interface. On the $\mathrm{CV}$ return scan the opposite process is proposed to take place; $\mathrm{FcMeOH}$ generated at the electrode is immediately oxidised back to $\mathrm{FcMeOH}^{+}$after donating an electron to the nanodiamond surface. For this mechanism to be feasible, the oxidation and reduction of nanodiamond surface functionalities must be at similar potentials to the standard potential of the solution redox probe. Electrode-immobilised $5 \mathrm{~nm}$ detonation nanodiamond has been shown to exhibit reversible redox peaks in this potential range, which shift potential with changing solution $\mathrm{pH} .{ }^{9}$ In addition, in situ IR studies have provided evidence that 'quinone-like' surface moieties on the nanodiamond are involved in the redox cycling. ${ }^{11}$ Although the $100 \mathrm{~nm}$ and $250 \mathrm{~nm}$ diamond particles used in this study are synthesised by different methods to the $5 \mathrm{~nm}$ nanodiamond and possess different morphologies, the observed current enhancements suggest similar surface species on each that can contribute to a catalytic reaction mechanism. All of the samples were oxidised prior to use and among the oxygen functionalities present it is likely that 'quinone-like' and 'hydroquinone-like' species are present on each to support the catalytic cycle in Fig. 1 b.

\subsection{Relationship between the particle size and the current enhancement}

In this study an inverse relationship between particle size and observed current enhancement was observed for both the oxidation of $\mathrm{FcMeOH}$ and reduction of $\mathrm{FcMeOH}^{+}$in the presence of 5, 10, 100 and $250 \mathrm{~nm}$ diameter nanodiamonds. The extent of the enhancement observed, quantified as the 'charge enhancement ratio', depends on the availability of nanodiamond surface functionalities to support the feedback mechanism. The concentration of these functionalities will depend on the nanodiamond particle size as well as the surface density of these groups. This latter factor will vary with oxidation pre-treatment, as well as the type of exposed crystal facets, edge sites and the defect density. A log-log plot of charge enhancement ratio with nanodiamond diameter produces a straight line, suggesting that a monomial power law relates these two properties. A straightforward relationship between the surface area to volume ratio of the particles and the charge enhancement ratio $(\sigma)$ should result from a $\sigma=3 r^{-1}$ fit to the data (where $r$ is the particle radius). However, a more complex relationship is found. Fractal scaling could be one explanation for the unusual relationship obtained, and it should also be noted that the $100 \mathrm{~nm}$ and $250 \mathrm{~nm}$ samples are not spherical, like the 5 and $10 \mathrm{~nm}$ samples. Most likely, the density of redox active sites on the different diamond samples differs. The highly active detonation nanodiamond surface is very defective and has much in common with amorphous carbons; it exhibits an incredibly diverse and dense coverage of functionalities. In contrast, the $100 \mathrm{~nm}$ and $250 \mathrm{~nm}$ samples are more 'diamondlike' in their character and thus the surface defect density and degree of $\mathrm{sp}^{2}$ bonding is much lower, resulting in fewer active sites. The $1000 \mathrm{~nm}$ diamond does not support the catalytic current enhancement (although neither does it block electrode activity). It is proposed that there are insufficient surface groups present on this material to produce a detectable catalytic current. The same mechanism is likely to take place at the surface of the $1000 \mathrm{~nm}$ diamond, however, the surface atom to bulk atom ratio is much smaller, so currents generated via this mechanism will be negligible in comparison to the diffusion controlled currents. 


\section{3. $\mathrm{pH}$ dependence of the current enhancement}

Current enhancement with $100 \mathrm{~nm}$ nanodiamond-modified electrodes was found to increase in the order $\mathrm{pH} 9<\mathrm{pH} 8<\mathrm{pH} 7<\mathrm{pH} 6<\mathrm{pH}$. The redox response of $\mathrm{FcMeOH}$ at the $\mathrm{BDD}$ electrode is independent of $\mathrm{pH}$, hence the ability of the nanodiamond to contribute to the catalytic cyclic must be the proton dependent step. $5 \mathrm{~nm}$ nanodiamond exhibits reversible redox peaks in voltammetry that shift by $c a .60 \mathrm{mV}$ to more negative potentials with each increase in $\mathrm{pH}$ unit. This is typical of a coupled reaction of one proton per electron transferred and consistent with a quinone/hydroquinone redox transformation, which in aqueous solution is a $2 \mathrm{H}^{+} / 2 \mathrm{e}^{-}$reaction. ${ }^{9}$ In the present study, we propose that similar surface species are present on the $100 \mathrm{~nm}$ nanodiamond and that the redox potentials of these functionalities are similarly $\mathrm{pH}$ dependent. The $\mathrm{pH} 5$ response suggests the potentials of the surface species are coincident with the potential of the $\mathrm{FcMeOH} / \mathrm{FcMeOH}^{+}$couple at this $\mathrm{pH}$. This match allows the most efficient electron transfer to take place between the nanodiamond and the solution species and hence the current enhancement is greater. As the $\mathrm{pH}$ is increased, the potential of the surface functionalities moves towards more negative potentials (according to Nernstian analysis of the proton dependence of the process), while that of the $\mathrm{FcMeOH} / \mathrm{FcMeOH}^{+}$couple is unchanged. As the potentials of the solution and surface species are not so well matched, the driving force for the catalytic process is decreased and the current enhancement is not so pronounced. Hence smaller current enhancements are noted at $\mathrm{pH} 9$.

\section{4. $\mathrm{pH}$ dependence of the magnitude of the off-set current}

Further evidence for $\mathrm{pH}$ dependent electron transfer between the nanodiamond surface groups and the $\mathrm{FcMeOH}$ species comes from inspection of the magnitude of the off-set reduction current at $0 \mathrm{~V}$ in Fig. 4. As described above, we attribute this current to the reduction of $\mathrm{FcMeOH}^{+}$present in the diffusion layer. The $\mathrm{FcMeOH}^{+}$is proposed to be generated by the spontaneous oxidation of $\mathrm{FcMeOH}$ at the nanodiamond (ND) surface in the absence of an applied potential:

$$
\mathrm{FcMeOH}+\mathrm{ND}_{\text {ox. }} \rightarrow \mathrm{FcMeOH}^{+}+\mathrm{ND}_{\text {red }}
$$

For such a process to be thermodynamically feasible, the nanodiamond surface functionalities must undergo reduction at a higher potential than the potential of the $\mathrm{FcMeOH} / \mathrm{FcMeOH}^{+}$couple. The magnitude of the reduction current for the $\mathrm{FcMeOH}^{+}$species suggests this is the case at $\mathrm{pH}<8$ with the largest driving force for the process occurring at the lower $\mathrm{pH}$ values. At $\mathrm{pH} 8$ and above the reduction currents are very small, indicating that electron transfer between the $\mathrm{FcMeOH}$ and the nanodiamond is negligible. This is consistent with the proton dependence of the potential of the surface functionalities. As $\mathrm{pH}$ increases, the potential shifts to more negative values and by $\mathrm{pH} 8$ is proposed to be found below that of the FcMeOH/ $\mathrm{FcMeOH}^{+}$couple, hence spontaneous electron transfer does not take place.

\subsection{Ionic strength dependence of the voltammetric response}

At low ionic strengths, a pre-peak to both the main oxidation and reduction waves becomes evident. This, along with the observed increased width of the 
voltammetric peaks in the presence of nanodiamond, as well as the decreased peak to peak separation ( $c a .30 \mathrm{mV}$ ), is diagnostic of adsorption of both FcMeOH and $\mathrm{FcMeOH}^{+}$at the electrode surface. As no evidence for adsorption is observed at the clean BDD electrode it is assumed that there is a strong affinity of the $\mathrm{FcMeOH}$ and $\mathrm{FcMeOH}^{+}$species for the nanodiamond surface. The positively charged $\mathrm{FcMeOH}^{+}$can clearly interact via electrostatic interactions with the negatively charged surface oxygen functionalities. Some current enhancement for the $\mathrm{Ru}\left(\mathrm{NH}_{3}\right)_{6}{ }^{3+}$ species at the $5 \mathrm{~nm}$ nanodiamond modified electrodes has previously been attributed to such an interaction. The strong adsorption of the neutral $\mathrm{FcMeOH}$ is more surprising as the nanodiamond surface is considered quite hydrophilic and less likely to interact with a largely hydrophobic molecule. However, the adsorption of hydrophobic species to oxidised nanodiamond has previously been reported and in fact exploited for the delivery of water-insoluble drugs. ${ }^{25}$ The $\mathrm{sp}^{2}$ bonding present at the nanodiamond surface provides ample opportunity for hydrophobic and $\pi-\pi$ stacking interactions with the aromatic pentacyclo- moieties of the $\mathrm{FcMeOH}$ molecule.

\subsection{Contribution of the adsorption processes to the enhanced current response}

The currents for FcMeOH oxidation at the nanodiamond-modified electrodes are much greater than observed previously for the $\mathrm{Fe}(\mathrm{CN})_{6}{ }^{4-}$ species. As the potential of the two species is similar, the same redox interactions with the nanodiamond surface groups are proposed. The difference in current enhancement is attributed to the extent to which the redox molecule can approach closely to the surface of the nanodiamond and the resulting efficiency of electron tunnelling between the two. The very negatively charged $\mathrm{Fe}(\mathrm{CN})_{6}{ }^{4-}$ is unlikely to be able to approach closely to the surface due to electrostatic repulsion, hence in the absence of any other factors it is not surprising that $\mathrm{FcMeOH}$ shows a higher current response that $\mathrm{Fe}(\mathrm{CN})_{6}{ }^{4-}$. However, the adsorption behaviour of $\mathrm{FcMeOH}$ and $\mathrm{FcMeOH}^{+}$is clearly a contributor to the very significant and unexpectedly large current enhancements we observe.

As stated above, FcMeOH undergoes spontaneous oxidation at the nanodiamond surface to generate $\mathrm{FcMeOH}^{+}$. This positively charged species will interact very strongly with the surface and become adsorbed. The strong interaction is illustrated by the affinity of the $\mathrm{FcMeOH}^{+}$for the nanodiamond surface, as found in the IR studies in Fig. 7. The contribution of the Gibbs energy of adsorption can shift the redox potentials of an adsorbed species relative to the solution species and results in the observation of pre-peaks in the CV. ${ }^{26,27}$ Here we propose that the surface adsorbed $\mathrm{FcMeOH}^{+}$also participates in the catalytic feedback mechanism, as shown in Fig. 8. Solution FcMeOH undergoes electron exchange with adsorbed $\mathrm{FcMeOH}^{+}$to generate solution $\mathrm{FcMeOH}^{+}$and adsorbed FcMeOH. The adsorbed FcMeOH can be re-oxidised to adsorbed $\mathrm{FcMeOH}^{+}$by the underlying BDD electrode, or by a redox process with the nanodiamond surface groups. Thus, in addition to the catalytic currents generated by the mechanism shown in Fig. 1b, the adsorbed species also mediate a catalytic process. The accumulative influence of both processes explains why currents are so significantly enhanced for this solution species compared to others previously studied. 
(1)

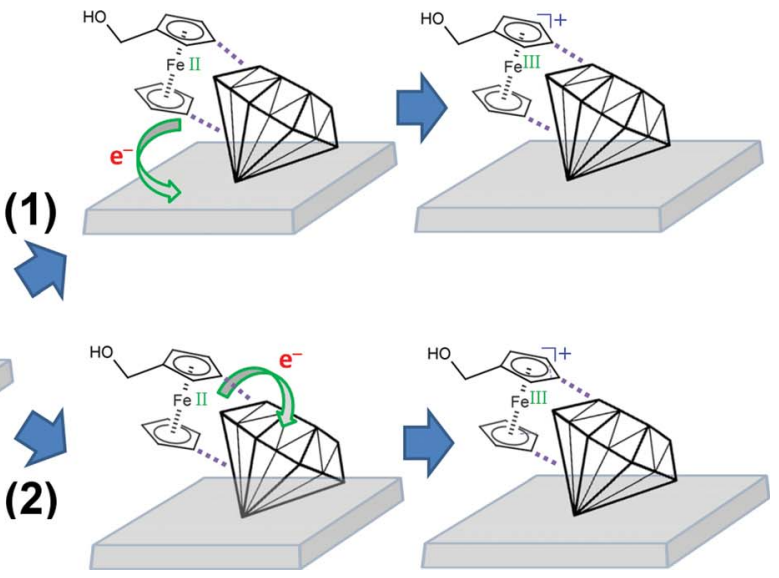

Fig. 8 Illustration of nanodiamond surface adsorbed $\mathrm{FCMeOH}^{+}$and its associated redox reaction. Re-oxidation of the $\mathrm{FCMeOH}$ product can then occur by either (1) the underlying electrode (when at sufficient potential) or (2) reaction with nanodiamond surface functionalities. The green arrows depict the direction of electron transfer.

\subsection{Scan rate dependence of the voltammetric response}

The scan rate dependence of the response illustrates further the different processes contributing to the catalytic currents. At slow scan rates, the pre-peak for the adsorption mediated process is very distinct from the main peak during the oxidation process. The two catalytic processes take place at distinct potentials; first via the adsorbed $\mathrm{FcMeOH}^{+}$species and then via the nanodiamond surface groups, indicating that adsorbed $\mathrm{FcMeOH}^{+}$is easier to reduce than the nanodiamond surface under these conditions. The factor determining the rate of the adsorption-mediated mechanism is the availability of adsorbed $\mathrm{FcMeOH}^{+}$to oxidise the solution $\mathrm{FcMeOH}$. Assuming the rate of regeneration of adsorbed $\mathrm{FcMeOH}^{+}$by reduction at the BDD electrode or nanodiamond surface is fast enough, the limit to this reaction is simply how much $\mathrm{FcMeOH}^{+}$is adsorbed at the nanodiamond interface. The adsorption-mediated process is so distinctly observed at slower scan rates because the flux of $\mathrm{FcMeOH}$ is smaller under these conditions, and hence mass transport of reactant can keep up with the rate of regeneration of the available $\mathrm{FcMeOH}^{+}$. At faster scan rates, the higher flux of solution FcMeOH leads to diffusion-controlled currents overwhelming the adsorption response and the pre-peak merges with the main diffusion-controlled peak. At higher scan rates and low concentrations a steady-state current begins to emerge (Fig. 6a). In this regime, we assume that catalysis takes place largely by the nanodiamond-mediated mechanism shown in Fig. 1b. The steady state current shows that the rate of $\mathrm{FcMeOH}^{+}$generation at the BDD electrode is exactly matched by the rate of $\mathrm{FcMeOH}^{+}$reduction by the nanodiamond surface functionalities. At slow scan rates a peak response is obtained for this process, as a thick diffusion layer containing $\mathrm{FcMeOH}^{+}$builds up and there are insufficient nanodiamond surface functionalities to maintain the catalytic cycle. At faster scan rates the diffusion layer is much thinner, so there is less $\mathrm{FcMeOH}^{+}$for the nanodiamond to reduce, hence the rate of electron transfer from the 
nanodiamond can keep up with the rate of $\mathrm{FcMeOH}^{+}$generation, leading to a steady state current response.

\section{Conclusions}

Modification of a BDD electrode with an immobilised layer of nanodiamond was found to enhance currents for the reversible oxidation of $\mathrm{FcMeOH}$. Current enhancement is inversely related to nanodiamond diameter, with enhancement increasing in the order $1000 \mathrm{~nm}<250 \mathrm{~nm}<100 \mathrm{~nm}<10 \mathrm{~nm}<5 \mathrm{~nm}$. In comparison to previous studies using negatively charged redox probes, we find that currents are much larger for the neutral species $\mathrm{FcMeOH}$ under the same experimental conditions. We attribute the current enhancement to two catalytic processes: i) electron transfer between the solution redox species and redox active groups on the nanodiamond surface; ii) electron transfer mediated by $\mathrm{FcMeOH}^{+}$ adsorbed onto the nanodiamond surface. The first process is $\mathrm{pH}$ dependent as it is believed that 'quinone-like' and 'hydroquinone-like' species on the nanodiamond surface mediate the redox process and thus it is coupled to proton transfer. The $\mathrm{FcMeOH} / \mathrm{FcMeOH}^{+}$redox cycle is more efficiently catalysed than the $\mathrm{Fe}(\mathrm{CN})_{6}{ }^{4-/ 3-}$ transformation reported previously, as the neutral and positive redox species are attracted rather than repelled by the negatively charged nanodiamond surface. The adsorption mediated process is observed most readily at slow scan rates and is due to electron self-exchange between adsorbed $\mathrm{FcMeOH}^{+}$ and $\mathrm{FcMeOH}$ in solution. $\mathrm{FcMeOH}^{+}$has a strong affinity for the nanodiamond surface, as confirmed by in situ ATR-FTIR experiments.

This study provides insight into the interaction of solution species with diamond and carbon surfaces under different physicochemical conditions. It also reveals mechanistic details of catalytic processes associated with the surfaces of nanodiamond materials. Catalytic currents within electrochemistry are of interest as they can allow energy intensive reactions to proceed at lower overpotentials (or allow a greater rate of reaction at the same overpotential). Such catalysts have applications in important sectors such as synthesis, energy conversion and molecular detection. Due to the particular mechanism of the nanodiamond electrocatalysis, nanodiamonds could find use within sensing devices, for example allowing detection of analytes at very low concentrations.

\section{Acknowledgements}

The authors would like to thank the EPSRC for funding this research (Grant No. EP/J010006/1). We also wish to extend our gratitude to Christoph Salzmann and Martin Rosillo-Lopez for use of their furnace and Steve Firth for assistance with TEM analysis.

\section{References}

1 For a review see: K. B. Holt, Electrochemistry of Nanodiamond Particles in Nanodiamond, ed. Oliver A. Williams, Royal Society of Chemistry, 2014, pp. 128-150.

2 K. B. Holt, C. Ziegler, D. J. Caruana, J. Zang, E. J. Millán-Barrios, J. Hu and J. S. Foord, Phys. Chem. Chem. Phys., 2008, 10, 303. 
3 K. B. Holt, Phys. Chem. Chem. Phys., 2010, 12, 2048.

4 W. Hongthani and D. J. Fermin, Diamond Relat. Mater., 2010, 19, 680.

5 J. B. Zang, Y. H. Wang, L. Y. Bian, J. H. Zhang, F. W. Meng, Y. L. Zhao, S. B. Ren and X. H. Qu, Electrochim. Acta, 2012, 72, 68.

6 J. B. Zang, Y. H. Wang, S. Z. Zhao, L. Y. Bian and J. Lu, Diamond Relat. Mater., 2007, 16, 16.

7 L. H. Chen, J. B. Zang, Y. H. Wang and L. Y. Bian, Electrochim. Acta, 2008, 53, 3442.

8 I. A. Novoselova, E. N. Fedoryshena, E. V. Panov, A. A. Bochechka and L. A. Romanko, Phys. Solid State, 2004, 46, 748.

9 K. B. Holt, D. J. Caruana and E. J. Millán-Barrios, J. Am. Chem. Soc., 2009, 131, 11272.

10 V. N. Mochalin, O. Shenderova, D. Ho and Y. Gogotsi, Nat. Nanotechnol., 2012, $7,11$.

11 J.-S. Tu, E. Perevedentseva, P.-H. Chung and C.-L. Cheng, J. Chem. Phys., 2006, 125, 174713.

12 J. Scholz, A. J. McQuillan and K. B. Holt, Chem. Commun., 2011, 47, 12140.

13 O. Shenderova, A. M. Panich, S. Moseenkov, S. C. Hens, V. Kuznetsov and H.-M. Vieth, J. Phys. Chem. C, 2011, 115, 19005.

14 A. Dementjev, K. Maslakov, I. Kulakova, V. Korolkov and V. Dolmatov, Diamond Relat. Mater., 2006, 15, 1813.

15 W. Zhao, J.-J. Xu, Q.-Q. Qiu and H.-Y. Chen, Biosens. Bioelectron., 2006, 22, 649.

16 J.-T. Zhu, C.-G. Shi, J.-J. Xu and H.-Y. Chen, Bioelectrochemistry, 2007, 71, 243.

17 S. Osswald, G. Yushin, V. Mochalin, S. O. Kucheyev and Y. Gogotsi, J. Am. Chem. Soc., 2006, 128, 11635.

18 A. Krueger, Chem.-Eur. J., 2008, 14, 1382.

19 I. Kiflawi, A. E. Mayer, P. M. Spear, J. A. Van Wyk and G. S. Woods, Philos. Mag. B, 1994, 69, 1141.

20 O. A. Williams, J. Hees, C. Dieker, W. Jäger, L. Kirste and C. E. Nebel, ACS Nano, 2010, 4, 4824.

21 M. Ozawa, M. Inaguma, M. Takahashi, F. Kataoka, A. Kruger and E. Osawa, Adv. Mater., 2007, 19, 1201.

22 J. P. Hsu and Y. C. Kuo, J. Colloid Interface Sci., 1997, 185, 530.

23 P. Debye and E. Hückel, Physikal. Z., 1923, 24, 185.

24 G. Socrates, Infrared characteristic group frequencies - Tables and charts, Wileyand Sons, $2^{\text {nd }}$ edn, 1994, pp. 94-156.

25 M. Chen, E. D. Pierstorff, R. Lam, S-Y. Li, H. Huang, E. Osawa and D. Ho, ACS Nano, 2009, 3, 2016.

26 R. H. Wopshall and I. Shain, Anal. Chem., 1967, 39, 1514.

27 R. H. Wopshall and I. Shain, Anal. Chem., 1967, 39, 1535. 